\title{
Lean Six Sigma en pequeñas y medianas empresas: un enfoque metodológico
}

\author{
Lean Six Sigma in small and medium enterprises: a methodological approach \\ Heriberto Felizzola Jiménez ${ }^{1} \quad$ Carmenza Luna Amaya² \\ Recibido 29 de julio de 2013, aceptado 17 de enero de 2014 \\ Received: July 29, 2013 Accepted: January 17, 2014
}

\begin{abstract}
RESUMEN
Six Sigma y Manufactura Esbelta son enfoques de mejora de la calidad y productividad que han sido implementados con gran éxito en grandes empresas a nivel mundial, en el ámbito de la manufactura y los servicios. Pero en la actualidad investigadores y expertos en el tema han encontrado hallazgos que evidencian dificultades en la implementación de este tipo de enfoques en pequeñas y medianas empresas (PYMES). Por esta razón, el presente artículo propone una metodología para la implementación de un enfoque integrado, comúnmente llamado Lean Six Sigma (LSS), el que se adapta a las necesidades y características de las PYMES. La metodología está compuesta de cuatro fases: donde la primera establece los factores claves en los cuales las PYMES deben prepararse para implementar LSS; en segundo lugar se plantea la identificación de focos de mejora y definición de un portafolio de proyectos; en tercer lugar, la ejecución de los proyectos priorizados; y por último, la evaluación de los resultados obtenidos. La metodología fue validada en una PYME dedicada a la fabricación de muebles de madera, donde la ejecución de los dos primeros proyectos LSS generaron importantes ahorros en costos de mala calidad, disminuciones en las devoluciones de productos, además de lograrse la implementación de buenas prácticas en la gestión de los procesos.
\end{abstract}

Palabras clave: Six Sigma, manufactura esbelta, PYMES, enfoque metodológico, Lean Six Sigma.

\begin{abstract}
Six Sigma and Lean Manufacturing are quality improvement and productivity approaches which have been implemented with great success in large global companies in the field of manufacturing and services. But now researchers and experts in the field have found that small and medium enterprises (SMEs) have difficulties in implementing such approaches. For this reason, this paper aims to develop a methodology for the implementation of an integrated approach, commonly called Lean Six Sigma (LSS), which is adapted to the needs and characteristics of SMEs. The methodology consists of four phases: the first phase establishes the key factors in which SMEs must be prepared to implement LSS, secondly the identification of improvement areas and definition of a project portfolio is set; as third phase, prioritized projects are executed, and finally, the results are evaluated. The methodology was validated in a SME dedicated to the manufacture of wooden furniture, where the execution of the first two LSS projects provided important savings in poor quality costs, reduction of product returns in addition to achieving the implementation of best practices in the process management.
\end{abstract}

Keywords: Six Sigma, lean manufacturing, SMEs, methodological approach, Lean Six Sigma.

\footnotetext{
1 Departamento de Ingeniería Industrial. Universidad de la Costa. Calle 58 Nº 55-66. Barranquilla, Colombia. E-mail: ing.heriberto.felizzola@gmail.com

2 Departamento de Ingeniería Industrial. Universidad del Norte. Km 5 Vía Puerto Colombia. Barranquilla, Colombia. E-mail: cluna@uninorte.edu.co
} 


\section{INTRODUCCIÓN}

LSS es un enfoque de mejora que ha tenido gran acogida gracias a su capacidad para dar solución efectiva a muchos de los problemas que enfrentan las organizaciones hoy [1]. Por esta razón, grandes empresas a nivel mundial han implementado este enfoque como una estrategia de negocios para mejorar la calidad de los productos y servicios, mejorar la eficiencia de los procesos, aumentar la satisfacción del cliente y aumentar la rentabilidad [2].

Si bien hay investigaciones que muestran que LSS puede generar beneficios en las organizaciones, independiente de su tamaño, hay otras que evidencian que estos beneficios se presentan en mayor grado en las grandes empresas en comparación con las PYMES [3-4]. Esta situación se debe en gran parte a factores como la falta de compromiso de la dirección; la limitación de recursos financieros y de personal; la resistencia al cambio; la ausencia de una cultura de mejora continua; la falta de liderazgo; la falta de reconocimiento de la importancia de la metodología; la falta de medición del desempeño de los procesos, entre otros [5-8].

En la última década investigadores, consultores y expertos en el tema han comenzado a estudiar factores críticos en la implementación de LSS en PYMES y a partir de allí han propuesto enfoques metodológicos [8-11]. Pero, aun el desarrollo teórico, conceptual y práctico es incipiente, por lo que muchas preguntas respecto de esta problemática no se han podido resolver con amplitud y claridad [10].

En este artículo se propone un enfoque metodológico para la implementación de LSS en PYMES, con lo que se pretende abordar aspectos como la falta de sistemas de medición, el uso de indicadores de gestión, la falta de alineación entre los proyectos LSS y los objetivos estratégicos; la falta de compromiso de la gerencia; la priorización de proyectos con alto impacto estratégico; la articulación de los proyectos con las necesidades y expectativas de los clientes; y la dificultad para ejecutar los proyectos LSS de forma sistemática, lo que garantice una correcta solución de las problemáticas abordadas.

En la primera parte del artículo se exponen los conceptos básicos de Six Sigma, Manufactura Esbelta y Lean Six Sigma; en la segunda parte se hace una revisión bibliográfica de los enfoques metodológicos y modelos para implementar LSS en PYMES; en la tercera parte se presenta la metodología propuesta; y por último se presentan los resultados obtenidos al aplicar la metodología en una empresa fabricante de muebles de madera.

\section{SIX SIGMA, MANUFACTURA ESBELTA Y LEAN SIX SIGMA}

Six Sigma es considerado como una evolución de las teorías clásicas de la calidad y la mejora continua, como el Control Estadístico de Proceso y la Administración de la Calidad Total TQM [12-13]. En este sentido Six Sigma toma algunos elementos de sus teorías precursoras y los estructura de forma sistemática, creando un enfoque mejorado y con mayor efectividad en la consecución de resultados, cuyo éxito se basa en los siguientes aspectos [14-17]:

- Se enfoca en los críticos de satisfacción del cliente (CTS);

- Se basa en la ejecución de proyectos de mejora;

- Hace uso intensivo de datos y herramientas estadísticas;

- Los resultados son medibles desde el punto operacional y financiero;

- Su efectividad en la consecución de resultados genera mayor compromiso de la gerencia y las personas;

- Los proyectos son desarrollados por personal capacitado en la metodología (cinturones negros, cinturones verdes o cinturones amarillos);

- Genera un cambio cultural orientado a la excelencia operacional.

Six Sigma está soportado en una metodología compuesta de cinco fases: Definir, Medir, Analizar, Mejorar y Controlar, comúnmente llamada DMAIC, por sus siglas en inglés (Define, Measure, Analize, Improve, Control), y tiene como objetivo aumentar la capacidad de los procesos, de tal forma que estos generen solo 3,4 defectos por millón de oportunidades (DPMO), con lo que los errores o fallas se hacen prácticamente imperceptibles para el cliente [5].

Esto ha llevado a muchas organizaciones a implementar Six Sigma como estrategia de negocios para aumentar su rentabilidad, mejorar la calidad 
de sus productos y servicios, llegando a mejorar su productividad y competitividad [2].

Por otro lado, la Manufactura Esbelta es un enfoque que permite mejorar la forma cómo la empresa organiza y gestiona la relación con sus clientes; la cadena de suministro; el desarrollo y la fabricación de sus productos, buscando generar mayores salidas con menores recursos [18]. La Manufactura Esbelta está soportada en cinco principios básicos, que son: identificar y definir el valor (Value); identificar los flujos de valor (Value Stream); alinear las acciones de la organización con los flujos de valor (Flow); permitir que las necesidades y expectativas del clientes jalonen (Pull); y por último, perseguir la perfección (Perfection).

Mediante estos principios, $\mathrm{y}$ de herramientas como el Justo a Tiempo (JIT), Kanban, 5S, Cambios Rápidos (SMED), el Mantenimiento Total Productivo, el Mapa de Flujo de Valor (Value Stream Mapping), los Poka Yokes, entre otros, se busca eliminar los desperdicios en sistemas de producción y servicios, los que se clasifican en: sobreproducción, inventarios, defectos, transportes, movimientos, reproceso y esperas [19].

Cada uno de estos enfoques hace un gran aporte a la mejora continua desde diferentes frentes; la disminución de defectos de forma estructurada en el caso de Six Sigma y el mejoramiento de los flujos de procesos e información por medio de la Manufactura Esbelta [20]. También es cierto que están soportadas en un marco común, como lo es la mejora de procesos, el enfoque al cliente, la formación especializada, el uso de metodologías estructuradas, la orientación a resultados operacionales y financieros, la gestión del cambio cultural, entre otros factores [21-22].

Este marco común facilita la implementación del enfoque integrado LSS, que presenta algunas ventajas, entre las que se cuenta: la integralidad y eficiencia con que se abordan diversos problemas organizacionales; evita la creación de estructuras paralelas para desarrollar procesos de mejora; permite crear un cultura de excelencia operacional centrada en la eficiencia y la satisfacción del cliente; y se amplía el foco de mejora de los proyectos con la creación de equipos multidisciplinarios y la vinculación de diferentes medidas de desempeño que apuntan a un objetivo más integral [23-25].

En la práctica Six Sigma y Manufactura Esbelta se articulan de diferentes formas, las que dependen del uso, modelos, principios, herramientas, medidas de desempeñó, estructuras de proyectos, entre otros [26]. Con ello se busca abordar problemáticas de diferente naturaleza relacionadas con la calidad, flujos de material, flujos de información y la generación de valor agregado.

\section{LEAN SIX SIGMA EN PYMES}

De acuerdo con el desarrollo y aplicación de LSS en las organizaciones, ha surgido la necesidad de adaptar este tipo de enfoques a las características de las PYMES [27]. Por esta razón, expertos en el tema han propuesto algunos enfoques metodológicos, pero alguno de estos presentan falencias, como suponer la disponibilidad de datos e información confiable; no considerar la importancia de los métodos de interacción con el cliente, los cuales permitan una retroalimentación constante; no toman en cuenta el liderazgo y el cambio cultural como un factor clave; no proponen estrategias específicas para superar las limitaciones financieras y estructurales de las PYMES; no se contempla la creación de redes con entidades públicas, organismos multilaterales, gremios, centros de investigación o instituciones académicas con el fin de superar los desafíos financieros, técnicos y tecnológicos que implica la implementación de LSS [10]. En la bibliografía se pueden encontrar varios modelos y metodologías que se han propuesto para implementar Six Sigma o LSS en PYMES, en la Tabla 1 se presenta un resumen de estos.

Para implementar LSS con éxito en una PYME se deben tener en cuenta muchos aspectos y factores críticos, entre los cuales se tiene: el compromiso de la dirección; la cultura organizacional; la articulación de LSS con la estrategia de la organización; el enfoque al cliente; la formación en LSS; las capacidades y competencias para la gestión y ejecución de proyectos; el nivel de conocimiento de la metodología y herramientas; la priorización de los proyectos LSS; y la capacidad financiera, técnica y tecnológica $[5,7,31,3]$. 
Tabla 1. Modelos y metodologías para implementar Six Sigma y LSS en PYMES.

\begin{tabular}{|l|l|}
\hline \multicolumn{1}{|c|}{ Autor } & \multicolumn{1}{c|}{ Enfoque/Contribución } \\
\hline $\begin{array}{l}\text { Thomas, Barton \& Chuke- } \\
\text { Okafor [28] }\end{array}$ & $\begin{array}{l}\text { Desarrollan un modelo para implementar de forma integrada Six Sigma y Manufactura } \\
\text { Esbelta. La metodología fue válida en una PYME, en la que se obtuvieron resultados } \\
\text { positivos en calidad, costos y tiempos de entrega. }\end{array}$ \\
\hline Chakravorty [29] & $\begin{array}{l}\text { Proponen un modelo que está compuesto por 6 fases que son: análisis estratégico; } \\
\text { identificación y formación de los equipos de mejora; identificación de las herramientas de } \\
\text { mejora; mapeo de la organización e identificación de oportunidades de mejora; desarrollo de } \\
\text { un plan detallado; y por último implementación, documentación y revisión del plan. }\end{array}$ \\
\hline $\begin{array}{l}\text { Knowlesa, Whickerb, } \\
\text { [30] }\end{array}$ & $\begin{array}{l}\text { Proponen un modelo para la implementación de Six Sigma en cadenas de suministro. } \\
\text { El modelo está formado por dos grandes ciclos, el primero se ocupa de los aspectos } \\
\text { estratégicos, como es la definición de objetivos y la medición del desempeño de la } \\
\text { organización, y el segundo ciclo está enfocado en la ejecución de los proyectos Six Sigma, } \\
\text { utilizando como base la metodológica DMAIC. }\end{array}$ \\
\hline $\begin{array}{l}\text { Kumar, Antony, \& Tiwari } \\
\text { [10] }\end{array}$ & $\begin{array}{l}\text { Desarrollan un marco lógico para la implementación de Six Sigma en PYMES, el que está } \\
\text { compuesto de 12 pasos divididos a su vez en 5 fases. Este marco brinda una guía paso a paso } \\
\text { para que las organizaciones puedan implementar Six Sigma, y va desde la preparación de la } \\
\text { organización hasta el sostenimiento a largo plazo. }\end{array}$ \\
\hline Thomas \& Barton [9] & $\begin{array}{l}\text { Proponen el uso de la metodología de auditoría en cadenas de suministro Quick Scan } \\
\text { (QS) como paso previo a la implementación de LSS en PYMES manufactureras. El QS es } \\
\text { utilizado en la fase de definición para identificar proyectos con impacto estratégico. }\end{array}$ \\
\hline $\begin{array}{l}\text { Murugesh, \& Sreenivasa } \\
\text { [11] }\end{array}$ & $\begin{array}{l}\text { Proponen un modelo para implementar con éxito LSS en PYMES. El modelo es denominado } \\
\text { DOLADMAICS (acrónimo de las palabras Deficiency Overcoming Lean Anchorage Define } \\
\text { Measure Analize Improve Control Stabilise) y permite la implementación de LSS en 5 } \\
\text { niveles. }\end{array}$ \\
\hline
\end{tabular}

\section{ENFOQUE METODOLÓGICO PROPUESTO}

Para estructurar la metodología que se propone en este artículo, primero se realizó una revisión de la bibliografía, con el fin de construir un marco de referencia sobre los factores críticos para implementar LSS en PYMES, también se identificaron estrategias y herramientas para abordarlos.

Habiendo identificado todo estos elementos, se diseñó una arquitectura básica de la metodología estructurada por fases, la que se comparó con otras metodologías para identificar falencias, fortalezas y aportes, luego se definieron en detalle cada una de las fases; con actividades y herramientas puntuales, por último se validó la metodología en una empresa pequeña dedicada a la fabricación de muebles de madera.

El enfoque metodológico está compuesto de cuatro fases, que en su orden son: Preparación, Identificación, Ejecución y Evaluación, todo esto soportado en una cultura de mejora Kaizen, un liderazgo enfocado en la mejora continua, cuya retroalimentación y dinamismo está impulsado por la visión, misión, los cambios en el entorno (competencia del mercado, marco regulatorio, necesidades de los clientes, entre otros factores) y la estrategia de la organización (ver Figura 1).

\section{METAS Y OBJETIVOS ESTRATÉGICOS}

Las metas y objetivos estratégicos demarcan el norte en la implementación de LSS, ya que por medio de estos las empresas definen iniciativas de mejora y fortalecimiento para sus procesos, productos y servicios.

Es importante que la empresa tenga claro cuáles son los indicadores de desempeño que marcan la evolución, el cumplimiento de las metas y objetivos, porque esto permite identificar y valorar el aporte de LSS a la organización [32].

\section{ELEMENTOS TRANSVERSALES}

Los elementos transversales en el marco metodológico son el motor para el despliegue de LSS en la organización, se puede decir que son elementos de la cultura organizacional que garantizan el avance y la continuidad; para que esto no se convierta en un proceso intermitente con esfuerzos aislados, cuya responsabilidad recae solo en el líder de despliegue. Este elemento se puede observar en la 


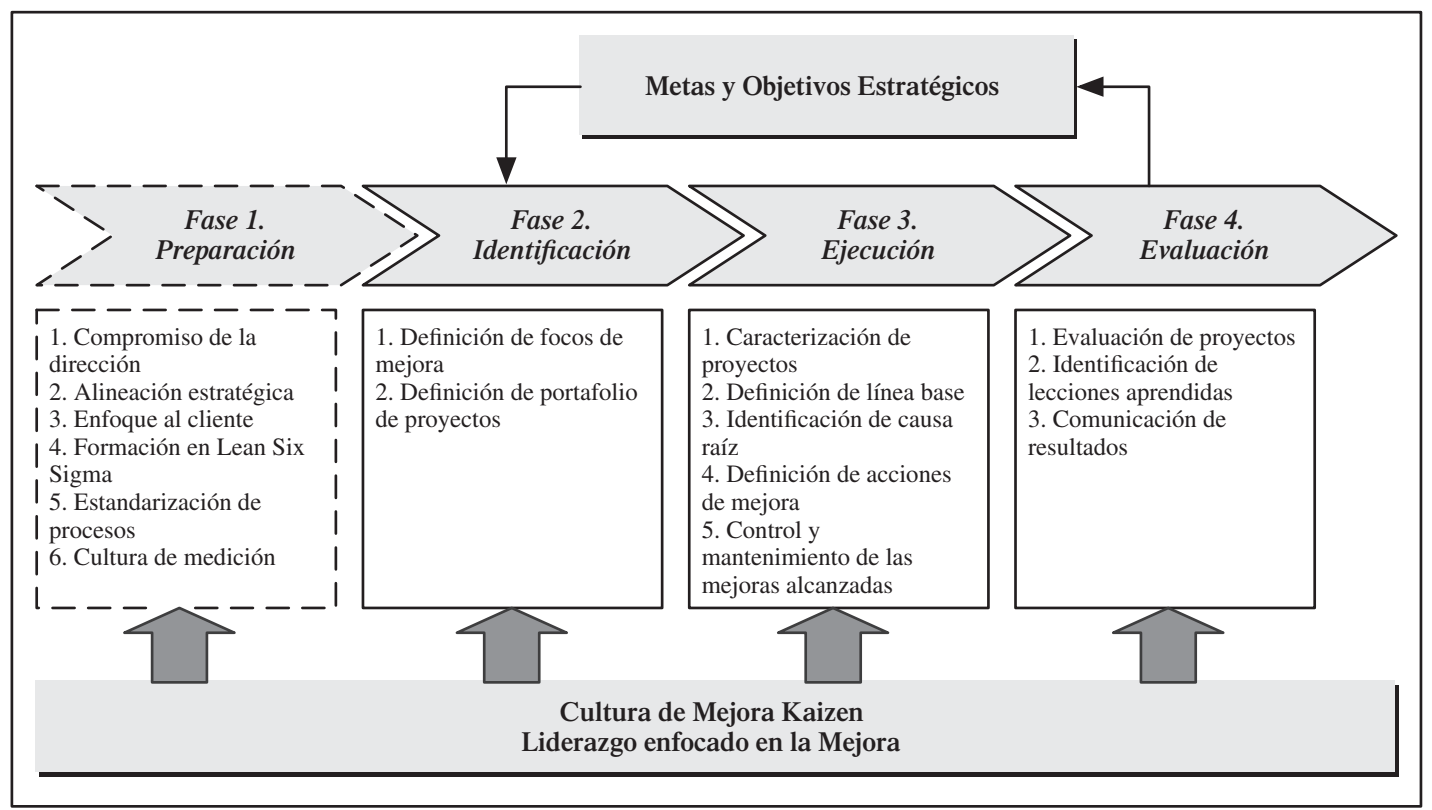

Figura 1. Metodología para implementar Lean Six Sigma en PYMES.

parte inferior de la Figura 1, y su descripción se presenta a continuación.

\section{Cultura de Mejora Continua Kaizen}

La resistencia al cambio es uno de los grandes retos que enfrentan las organizaciones cuando se llevan a cabo proyectos LSS [33-34]. Por esta razón, es necesario lograr que las personas incluyan dentro de su rutina diaria el componente de mejora continua Kaizen, que busca introducir cambios pequeños y graduales de forma constante, más que en grandes cambios proyectados en largos periodos [35].

\section{Liderazgo Enfocado en la Mejora}

Los cambios en la organización requieren una gestión constante y efectiva, ya que las personas necesitan ser motivadas para introducir mejoras en los procesos y conseguir resultados, pero esta responsabilidad es mayormente de la alta dirección, debido a que son los encargados de liderar e impulsar los cambios desde la planeación hasta la ejecución [36].

\section{FASE 1. PREPARACIÓN}

En esta fase la organización debe emprender una serie de acciones que garanticen el cumplimiento de aquellas características o condiciones necesarias para implementar con éxito la metodología. Con esto se busca crear las condiciones mínimas necesarias para desarrollar proyectos LSS con alta probabilidad de éxito. Una descripción detallada de cada una de las condiciones necesarias se suministra a continuación.

\section{Compromiso de la Dirección}

El compromiso de la dirección es clave dentro de todo el proceso, ya que es uno de los factores que garantiza la continuidad de LSS, esto, mediante la asignación de los recursos necesarios y el impulso en toda la organización [37].

\section{Alineación Estratégica}

Para definir el rumbo y enfoque que debe tomar LSS dentro de la organización, primero se debe conocer su planeación estratégica, y en caso de no tenerla, se debe por lo menos conocer las proyecciones de la organización a mediano y largo plazo [38].

\section{Enfoque al cliente}

Es importante que la organización conozca quiénes son las partes interesadas (Stakeholders) en este proceso, y una de ellas son los clientes. Por esta razón es necesario identificar quiénes son, cuáles son su características, cómo están segmentados y cuáles son sus necesidades [32].

Algunas de las herramientas que se pueden utilizar son: el modelo de Kano, el Análisis de CTS, el 
Despliegue de la Función Calidad QFD, el SIPOC, entre otros [39, 19].

\section{Formación en LSS}

Toda persona que participa en un proyecto LSS, sea liderando, coordinando, ejecutando o apoyando los proyectos, debe recibir formación en las metodologías y herramientas LSS, ya que esto contribuye a la correcta ejecución de los proyectos y a un mejor desempeño del personal [36].

Para el caso de las PYMES, la intensidad horaria y estructura del programa de formación puede variar respecto de las grandes empresas, pero esto dependerá de la capacidad financiera, la disponibilidad de tiempo del personal que participa y las necesidades específicas de acuerdo con el tipo de empresa (manufactura o servicios) [31].

\section{Estandarización de los procesos}

Contar con un proceso estandarizado y estadísticamente estable, es una de las condiciones de partida en todo proyecto LSS, ya que con esto se puede establecer una línea base, hacer una gestión efectiva de los procesos y evidenciar las mejoras alcanzadas con los proyectos $[31,40]$.

\section{Cultura de Medición}

La medición del desempeño en los procesos por medio de indicadores, es fundamental en toda marcha de mejora, y más aún en proyectos LSS, ya que brinda un conocimiento real acerca del progreso y los resultados alcanzados $[4,6]$.

\section{FASE 2. IDENTIFICACIÓN}

En la fase de identificación la organización debe decidir cuál será el enfoque de los proyectos LSS. Dos preguntas básicas a resolver en esta fase son: ¿Qué se quiere lograr con la implementación de LSS? [41] y ¿Qué proyectos se deben ejecutar para lograr los objetivos estratégicos? [42]. Por tanto, primero se deben definir los focos de mejora y luego se debe crear un portafolio de proyectos, del cual saldrán los proyectos a ejecutar. A continuación se presenta una descripción de estas etapas.

\section{Etapa 1. Identificación de los Focos de Mejora}

Debido a las limitaciones financieras, de recursos e infraestructura en las PYMES, se debe definir previamente sobre qué áreas, procesos y productos/ servicios críticos se debe trabajar para la consecución de los objetivos estratégicos, y cuáles serán los indicadores de gestión que se utilizarían para medir su desempeño. En virtud de que la identificación de las áreas de mejora tiene un alto componente estratégico, es necesaria la participación de los directivos de la organización.

\section{Etapa 2. Definición de un Portafolio de Proyectos} Luego de haber identificado los focos de mejora, se define un portafolio de proyectos LSS, con lo que se busca fortalecer las áreas de las organizaciones identificadas como claves.

Se debe evitar desarrollar proyectos que no estén articulados con los focos de mejora, ya que se corre el riesgo de desperdiciar tiempo y recursos en acciones de mejora que no están alineadas con la estrategia de la organización, no generan un alto impacto en los resultados financieros, no contribuyen significativamente con la satisfacción del cliente y por tanto no generan el compromiso de la dirección. Para construir el portafolio de proyectos se debe:

1. Identificar posibles proyectos.

2. Evaluar los proyectos previamente identificados; teniendo en cuenta factores como el tiempo de duración, inversión, nivel de conocimiento de las causas, complejidad, impacto financiero, impacto en el cliente, entre otros [43].

3. Priorizar los proyectos de acuerdo con la calificación obtenida en la evaluación

\section{FASE 3. EJECUCIÓN}

Ya preparada y con proyectos seleccionados, la organización puede proceder a la ejecución. En esta fase la organización ejecuta y hace seguimiento a los proyectos previamente seleccionados, para esto se utiliza como base la metodología DMAIC de Six Sigma y las herramientas de la Manufactura Esbelta. En la Figura 2 se presenta el proceso genérico para la ejecución de un proyecto LSS.

Etapa 1. Caracterización de los Proyectos (Definir) En esta etapa se busca definir los aspectos generales del proyecto, tales como: título del proyecto, objetivo, planteamiento del problema, equipo de trabajo, programación de las actividades, métricas, 


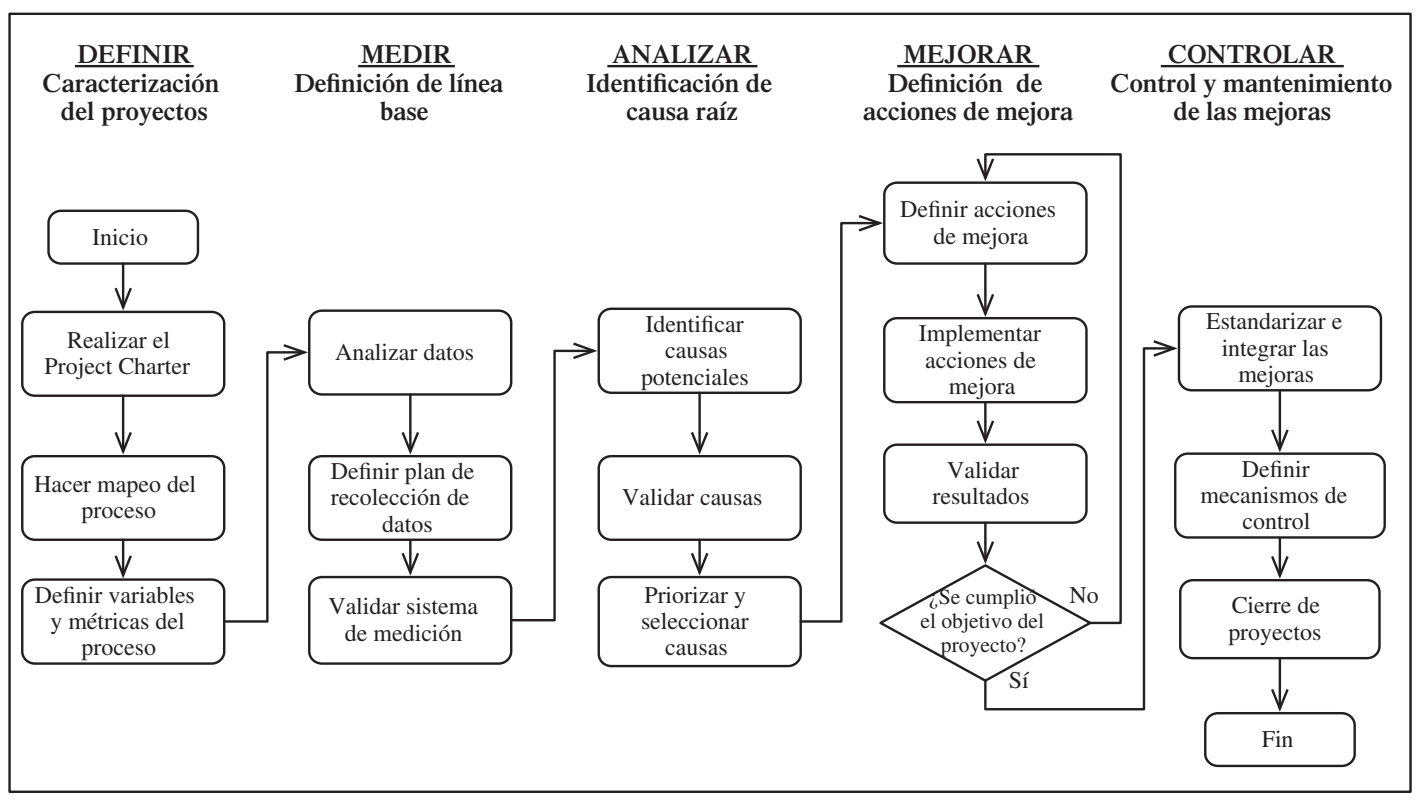

Figura 2. Metodología DMAIC para Proyecto Lean Six Sigma en PYMES.

variables del proceso, actividades del proceso $\mathrm{u}$ otra información necesaria para realizar completa caracterización del proyecto. El desarrollo de la fase comprende básicamente tres (3) actividades:

1. Realizar el Project Charter: la información contenida en el Project Charter varía de acuerdo con el nivel de detalle que la organización requiera, pero como mínimo debe contener: título del proyecto objetivo, planteamiento del problema, estado actual de las métricas LSS, ahorro proyectado y equipo del proyecto.

2. Hacer un mapeo del proceso y definir variables: antes de emprender cualquier acción se debe analizar a profundidad el proceso a intervenir y sus respectivas variables. En esta actividad se pueden utilizar herramientas como los flujogramas, mapas de procesos, SIPOC o el Mapa de Flujo de Valor.

3. Identificar métricas del proceso: basado en el análisis que se haga del proceso, se identificarán las medidas de desempeño claves sobre las cuales se hará seguimiento durante y después de la ejecución del proyecto.

\section{Etapa 2. Definición de Línea Base (Medir)}

Una de las características fundamentales del enfoque LSS es el uso intensivo de datos e información, utilizados para el análisis de los procesos, el diseño de estrategias de mejora y la toma de decisiones. Por esta razón, antes desarrollar las fases de análisis y mejora es necesario asegurar que las fuentes de información y los sistemas de medición sean lo suficientemente confiables, para evitar tomar acciones erróneas que conlleven a la falta de resultados. Adicionalmente, en esta etapa se debe levantar una línea base, que sirva como un punto de partida para evaluar la efectividad de las mejoras alcanzadas con la consecución de cada proyecto. Las actividades en esta etapa son:

1. Validar sistema de medición: un sistema de medición debe ser evaluado con los siguientes criterios: exactitud (Sesgo), linealidad, estabilidad, repetibilidad, reproducibilidad y sensibilidad $[19,44]$. Según el tipo de dato se pueden utilizar diferentes métodos estadísticos: Análisis R\&R y Estudio de Linealidad y Sesgo para datos continuos; y Análisis de Concordancia, cuando se evalúan atributos.

2. Definir plan de recolección de datos: se debe diseñar un plan de recolección de datos basado en los conceptos de muestro, esto con el fin de recopilar información necesaria para hacer seguimiento a las medidas de desempeño y realizar análisis estadístico que permita identificar la causa raíz. 
3. Definir línea base: se debe definir el estado inicial del proceso, producto o servicios, mediante un análisis de capacidad de proceso y de los cálculos de métricas LSS como el Nivel Sigma, DPO, DPU, DPMO, RTY, Takt Time y Lead Time.

\section{Etapa 3. Identificación de causa raíz (Analizar)}

Para identificar la causa raíz primero se deben identificar las causas potenciales; en segundo lugar las causas deben ser validadas con la ayuda de métodos estadísticos y análisis por los equipos de trabajo; y por último se deben definir las causas que tienen mayor impacto sobre el problema. A continuación presentan las actividades claves:

1. Identificar causas potenciales: para esto se pueden utilizar herramientas como el diagrama de afinidad, diagrama de Isikawa y Análisis de Modo y Efecto de Fallo (AMEF).

2. Analizar y validar causas: para esto se pueden utilizar herramientas como las pruebas de hipótesis, el Análisis de Varianza (ANOVA), el análisis de correlación, los Diseños de Experimentos (DOE), métodos estadísticos no paramétricos y los métodos estadísticos multivariados.

3. Priorizar y seleccionar causas a trabajar: luego de validadas las causas, se deben priorizar de acuerdo con la contribución que estas tengan sobre el problema o la variabilidad del Proceso, para esto se pueden utilizar herramientas como la matriz causa-efecto y el AMEF.

\section{Etapa 4. Definición de acciones de mejora (Mejorar)}

Basada en la causa raíz identificada en la etapa anterior, se deben definir acciones específicas para darle solución al problema y alcanzar el objetivo propuesto con el desarrollo del proyecto. Las soluciones planteadas pueden ser acciones de rápido cumplimiento o también pueden conllevar a la implementación de un conjunto de acciones basadas en buenas prácticas de gestión. En este punto las herramientas de la Manufactura Esbelta juegan un papel fundamental, ya que permiten analizar y diseñar soluciones. Al final, todas las acciones se deben consolidar en un plan piloto que permita hacer seguimiento y control. Las actividades claves en esta etapa son:
1. Definir acciones de mejora: para esto se pueden utilizar herramientas estadísticas y de gestión, no solo para generar ideas y soportar decisiones, sino también para estructurarlas en un plan de acción con actividades concretas. Para esto se pueden utilizar métodos estadísticos, como el análisis de regresión, DOE, los métodos de superficie de respuesta, la simulación, y también se pueden utilizar herramientas de la Manufactura Esbelta, como las 5S, el Kanban, los Flujos de una Pieza, el Mantenimiento Total Productivo, el SMED, la Gerencia Visual, el Balanceo de Líneas y los Poka Yokes.

2. Implementar acciones de mejora: las acciones de mejora se deben implementar de acuerdo con el plan previamente definido, y sobre el cual se debe hacer un seguimiento periódico para verificar su cumplimiento y tomar acciones correctivas cuando sea necesario.

3. Validar resultados: en la medida que se van implementando las acciones de mejora, se debe evaluar el impacto que estas generan sobre el problema, esto se hace con la revisión y análisis de las métricas LSS, métricas operacionales y métricas financieras. Si no se cumple el objetivo se deben revisar y replantear las acciones definidas.

\section{Etapa 5. Control y mantenimiento (Controlar)}

En este punto del proyecto se busca incorporar y estandarizar los cambios introducidos en la etapa de mejora. Por esta razón es importante documentar los procesos o procedimientos modificados, y para esto se puede utilizar el enfoque de aseguramiento de la calidad a través de la ISO 9001.

Adicionalmente se deben diseñar mecanismos para garantizar que los cambios y mejoras alcanzadas se mantengan a lo largo de tiempo, con el fin de darle continuidad más allá del cierre del proyecto. Las actividades claves en esta etapa son:

1. Estandarizar e integrar las mejoras a los procesos: en esta actividad se deben levantar manuales de procedimientos, diagramas de procesos, mapas de procesos, y si la empresa cuenta con un sistema de gestión de calidad, entonces debe actualizar el manual de calidad. 
2. Definir mecanismos de control de mejoras: se deben diseñar mecanismos para hacer seguimiento y mantener las mejoras alcanzadas.

Por esta razón es necesario comunicar los cambios a las partes interesadas, y si es necesario capacitarlos, para asumir los nuevos cambios. Para crear disciplina y orden se puede acudir a herramientas como las 5S, y para monitorear indicadores se pueden utilizar los Gráficos de control y la Gerencia Visual.

3. Cerrar proyectos: cuando se han alcanzado las metas e impactos esperados se debe elaborar un informe de cierre de proyecto, mediante el cual se pueda comunicar a las partes interesadas, de forma clara y precisa, todos los resultados del proyecto.

\section{FASE 4. EVALUACIÓN}

La evaluación de los resultados derivados de la ejecución del portafolio de proyectos es fundamental, ya que permite identificar nuevas oportunidades de mejora y lecciones aprendidas que conlleven a la realización de nuevos proyectos LSS. Es importante que el equipo LSS pueda destacar los aspectos positivos y negativos de cada proyecto, ya que esto enriquece el aprendizaje organizacional [10]. Algunas actividades claves en la fase de evaluación son:

1. Evaluar resultados de los proyectos: se deben evaluar todos los proyectos ejecutados y verificar su impacto en los resultados globales de la organización. Estos resultados se deben evaluar teniendo en cuenta los siguientes aspectos: grado de cumplimiento de los objetivos, impacto financiero, impacto en los productos o servicios, impacto en la eficiencia y productividad de los procesos e impacto en la satisfacción de los clientes.

2. Levantar lecciones aprendidas: las lecciones se definen de acuerdo con la retroalimentación y evaluación de cada proyecto, con el fin de replicar buenas prácticas en la ejecución de proyectos LSS y evitar la reincidencia en equivocaciones o errores de anteriores proyectos, esto al final es un activo de conocimiento y experiencia en LSS derivado del aprendizaje organizacional.

\section{RESULTADOS DE LA IMPLEMENTACIÓN EN UNA PYME}

La validación de la metodología se llevó a cabo en una empresa pequeña dedicada a la fabricación de muebles de madera. Teniendo en cuenta que la empresa hasta el momento de la validación no contaba con un direccionamiento estratégico, se procedió a identificar las metas a mediano y largo plazo, algunas de estas son: posicionar sus productos entre los de mayores ventas; mejorar los estándares de calidad de sus productos, lo que se refleje en la disminución de los servicios generados a causa de devoluciones por parte del cliente final; mejorar la productividad de sus procesos de producción, lo que impacte en los costos de producción, los costos de mala calidad, los reprocesos, los tiempos de ciclo y la capacidad de producción.

\section{Fase 1. Preparación}

Esta primera fase se desarrolló con la participación de la empresa en un programa de fortalecimiento de PYMES por medio de LSS financiado por el Banco Interamericano de Desarrollo (BID). Con el apoyo financiero y técnico de este programa se logró capacitar al gerente y a varios empleados de la organización, con lo que se pudieron abordar dos aspectos claves, como son el Compromiso de la Dirección y la Formación para LSS.

Por otro lado se desarrollaron algunas acciones para abordar los cuatro (4) aspectos restantes, alguna de estas acciones son:

1. Alineación estratégica de LSS: teniendo en cuenta que la empresa no contaba con una planeación estratégica, se tomó la decisión de construir un Mapa Estratégico, buscando definir acciones que se puedan abordar a partir de la implementación de LSS.

2. Enfoque al cliente: para la identificación de las necesidades y expectativas de los clientes se utilizó la herramienta SIPOC y se complementó con una identificación de los Críticos de Satisfacción del Cliente (CTS). Algunos críticos identificados son: estética del producto, resistencia, durabilidad de la pintura, variedad del diseño, tiempo de entrega y el costo del producto.

3. Estandarización de procesos: se realizó un diagnóstico basado de las técnicas del estudio del trabajo y se diseñó un plan de mejora basado en la filosofía de las $5 \mathrm{~S}$.

4. Cultura de medición: en vista de la necesidad de implementar procesos de medición en cada 
una de las etapas del proceso de producción, se diseñó un instrumento para recolectar datos de las devoluciones internas en las diferentes etapas del proceso de fabricación, y basado en esto se construyeron indicadores de calidad, sobre los cuales se hizo monitoreo y control.

\section{Fase 2. Identificación}

La identificación de los focos de mejora en la empresa se hizo desde dos perspectivas, defectos y productos. La primera con la identificación de las no conformidades sobre las cuales los clientes tenían mayores quejas, y la segunda sobre los productos en los cuales se presentaba la mayor cantidad de devoluciones o reclamos de garantía. Para esto se tomaron los registros de las devoluciones y se realizó un análisis de Pareto para cada perspectiva.

Los resultados de análisis mostraron que el $80 \%$ de los defectos estaba concentrado en cuatro (4) tipos de defectos (ver Figura 3): problemas en pintura, problemas debido al secado de la madera, problemas en el armado y problemas por el embalaje del producto.

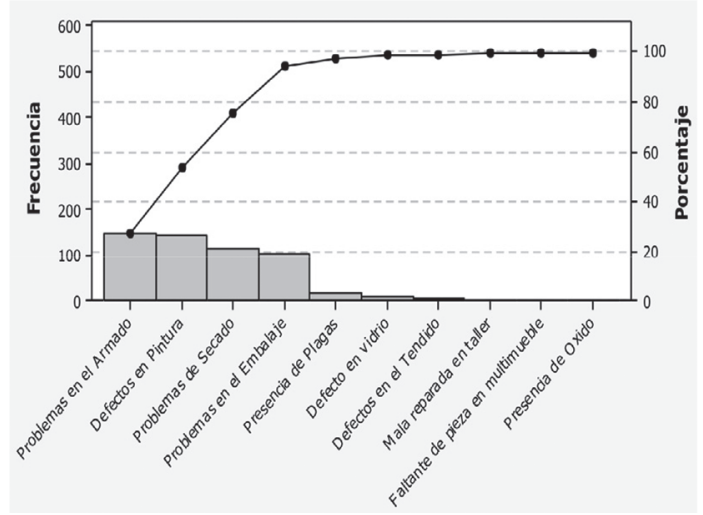

Figura 3. Pareto para devoluciones por tipo de defecto.

Por lo anterior tomó la decisión de concentrar los esfuerzos en estos pocos factores y a la vez obtener un impacto considerable en la reducción de las devoluciones y por ende la calidad del producto. En la Tabla 2 se presentan los focos de mejora definidos y los proyectos identificados.

\section{Fase 3. Ejecución}

De los siete (7) proyectos identificados se priorizaron y ejecutaron dos proyectos. A continuación se presenta el desarrollo de cada una de las fases del DMAIC para el proyecto: Disminución de las devoluciones de gavetas defectuosas.

Tabla 2. Identificación de focos de mejora y proyectos.

\begin{tabular}{|l|l|}
\hline \multicolumn{1}{|c|}{$\begin{array}{c}\text { Focos de } \\
\text { mejora }\end{array}$} & \multicolumn{1}{c|}{ Proyectos identificados } \\
\hline $\begin{array}{l}\text { Defectos en } \\
\text { pintura }\end{array}$ & $\begin{array}{l}\text { 1. Disminución de las devoluciones de } \\
\text { productos por problemas en el acabo de } \\
\text { la pintura } \\
\text { 2. Disminución de las devoluciones de } \\
\text { productos por presencia de manchas en } \\
\text { la pintura }\end{array}$ \\
\hline $\begin{array}{l}\text { Problemas } \\
\text { de secado de } \\
\text { la madera }\end{array}$ & $\begin{array}{l}\text { 3. Disminución de las devoluciones de } \\
\text { productos por madera rajada }\end{array}$ \\
\hline $\begin{array}{l}\text { Problemas } \\
\text { en el armado }\end{array}$ & $\begin{array}{l}\text { 4. Disminución de los reprocesos por } \\
\text { mala colocación de herrajes } \\
\text { 5. Disminución de las devoluciones de } \\
\text { alcobas por pegas abiertas en largueros, } \\
\text { pieceros y cabeceras de las camas } \\
\text { 6. Disminución de las devoluciones de } \\
\text { gavetas defectuosas }\end{array}$ \\
\hline $\begin{array}{l}\text { Problemas } \\
\text { en el } \\
\text { embalaje de } \\
\text { productos }\end{array}$ & $\begin{array}{l}\text { 7. Disminución de las devoluciones de } \\
\text { productos por presencia de rayones en la } \\
\text { superficie }\end{array}$ \\
\hline
\end{tabular}

Definir: los procesos de corte y armado son los encargados de garantizar que las gavetas posean las especificaciones requeridas, para un correcto acople en los nocheros, tocadores y pieceros de algunas camas. Al inicio del proyecto la empresa había identificado que las devoluciones por defectos en las gavetas estaba en $15,59 \%$, lo que se considera bastante crítico, por los costos que esto representa en cuanto a tiempo y material desperdiciado. Los costos de mala calidad por cada gaveta defectuosa es de $\$ 14.000$ y esto representa un costo promedio mensual de $\$ 4.914 .000$. En esta primera fase del proyecto se realizó un análisis del proceso de corte de madera y armado de las gavetas, con el fin de definir las variables claves que sirvan como base para identificar la causa raíz del problema.

Medir: teniendo en cuenta que la identificación de las gavetas defectuosas se hace con una inspección visual, se procedió a realizar un estudio de concordancia de atributos, cuyos resultados arrojaron una tasa de error general del $13,3 \%$, con lo que se consideró que la consistencia en el proceso y los criterios de inspección eran aceptables. Después de esto se verificó que el proceso estuviera bajo control 


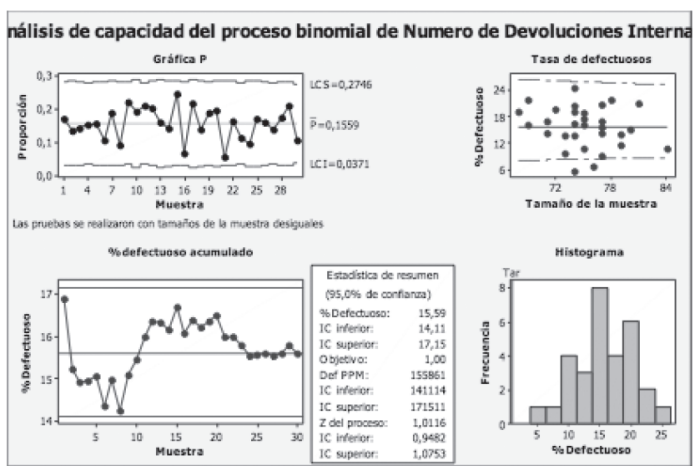

Figura 4. Análisis de Capacidad Binomial.

estadístico y se realizó un estudio de capacidad de proceso binomial (variable pasa/no pasa). Los resultados arrojaron un DPMO de 155.861 y un nivel sigma de 2,52 (ver Figura 4).

Analizar: para la identificación de las causas raíces de las gavetas defectuosas se hizo un análisis de las variables involucradas en los procesos de corte y armado. En el proceso de corte se identificaron las causas por la cuales la madera cortada no cumplía con las tolerancias establecidas, ya que este problema dificulta el acople de las gavetas con los nocheros y tocadores de las alcobas. Por otro lado, en el proceso de armado se identificaron las causas por las cuales las gavetas quedaban mal ensambladas. Se utilizó el diagrama de Isikawa como herramienta para identificar cada una de las causas, las cuales se clasificaron en seis (6) categorías: Mano de obra, Materiales, Métodos, Máquinas, Mediciones y Ambiente de trabajo.

Las causas asociadas a factores humanos se validaron según exploración de varianza y los factores procedimentales por el análisis de modo y efecto de fallo (AMEF). Los resultados mostraron que la causa raíz estaba asociada mayormente a errores en el proceso de corte, en cambio la experiencia y conocimiento del operario no eran relevantes. Se pudo establecer que los procesos de corte no estaban debidamente estandarizados, no se utilizaban guías o instrumentos de apoyo para realizar un corte de alta precisión y los procesos de inspección en el área de corte no permitían garantizar una calidad aceptable para el proceso de ensamble.

Mejorar: teniendo en cuenta los resultados de la fase de análisis se elaboró un plan que incluye las siguientes acciones de mejora:

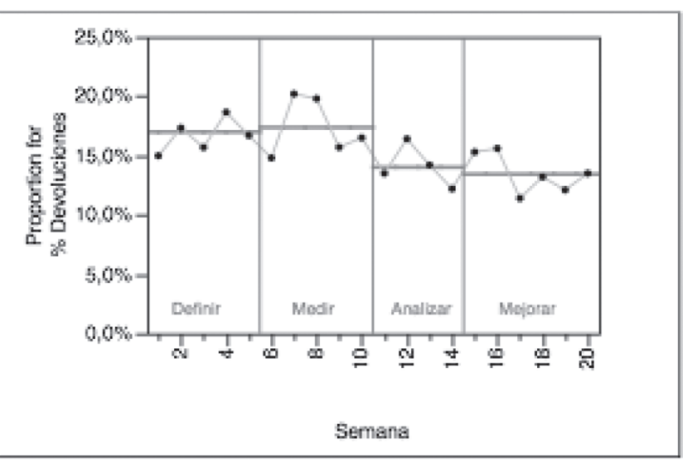

Figura 5. Comportamiento del porcentaje de devoluciones

- Diseñar y estandarizar el proceso de corte;

- Diseñar e implementar guías y dispositivos de apoyo al proceso de corte y armado;

- Capacitar y entrenar a los operarios en el nuevo proceso de corte y el uso de guías y dispositivos;

- Diseñar un proceso eficiente de verificación de dimensiones de las piezas cortadas.

Con la implementación de estas acciones se logró una reducción en los defectos de 15,59\% a 13,52\% (ver Figura 5). Esto también generó una disminución en el DPMO, pasando de 155.861 a 102.000 , y se aumentó el nivel sigma de 2,52 a 2,74.

Controlar: habiendo alcanzado mejoras importantes en el proceso de corte y armado de gavetas, y con el fin de mantenerlas, se implementaron las siguientes acciones:

- Utilizar una gráfica de control P, para hacer seguimiento al porcentaje de devolución de gavetas defectuosas;

- Realizar auditorías mensuales, para hacer seguimiento sobre el problema de gavetas defectuosas, las posibles causas y las mejoras alcanzadas;

- Implementar la gerencia visual en el área de armado para que el jefe de producción y los operarios del área puedan hacer seguimiento constante sobre los métricos relevantes del área.

\section{Fase 4. Evaluación}

En esta fase se evaluaron los resultados de los proyectos, con lo que se pudieron identificar los siguientes impactos: 
- Mejoras en el nivel de calidad de las alcobas, con la reducción de los problemas por pegas abiertas y gavetas defectuosas;

- Aumento en la capacidad del proceso de corte, específicamente en la exactitud de las dimensiones de las piezas cortadas;

- Aumento en la capacidad del proceso de armado de gavetas, ya que se disminuyó el porcentaje de no conformidad en esta área;

- Aumento en la capacidad del proceso de armado de camas, ya que se disminuyó el porcentaje de no conformidad por presencia de pegas abiertas;

- Menores quejas por parte del cliente externo, respecto de la presencia de pegas abiertas y gavetas defectuosas.

En la Tabla 3 se presenta un resumen de los resultados en los proyectos ejecutados.

Tabla 3. Resultado de los proyectos.

\begin{tabular}{|l|l|l|}
\hline & \multicolumn{1}{|c|}{ Proyecto 1 } & \multicolumn{1}{c|}{ Proyecto 2 } \\
\hline Título & $\begin{array}{l}\text { Disminución de } \\
\text { las devoluciones } \\
\text { por pegas abiertas }\end{array}$ & $\begin{array}{l}\text { Disminución de las } \\
\text { devoluciones por } \\
\text { gavetas defectuosas }\end{array}$ \\
\hline Objetivo & Reducirlas 1\% & Reducirlas al 1\% \\
\hline Métrica & $\begin{array}{l}\text { Antes: } 20,44 \% \\
\text { Después: } 11,71 \%\end{array}$ & $\begin{array}{l}\text { Antes: } 15,59 \% \\
\text { Después: 10,20\% }\end{array}$ \\
\hline Nivel sigma & $\begin{array}{l}\text { Antes: } 2,3 \\
\text { Después: } 2,7\end{array}$ & $\begin{array}{l}\text { Antes: 2,51 } \\
\text { Después: } 2,75\end{array}$ \\
\hline DPMO & $\begin{array}{l}\text { Antes: } 204.338 \\
\text { Después: } 117.100\end{array}$ & $\begin{array}{l}\text { Antes: } 151.861 \\
\text { Después: } 102.000\end{array}$ \\
\hline Ahorro & \$18.000.000* & \$20.000.000* \\
\hline
\end{tabular}

*Expresado en pesos colombianos.

De las lecciones aprendidas identificadas en el proceso de evaluación se destaca el trabajo a profundidad que se debe hacer sobre el factor humano antes de implementar cualquier cambio en sus métodos de trabajo, esto incluye sensibilización, capacitación, motivación, participación e incentivos. Esto se debe a que el factor humano limitó en gran manera la consecución de los objetivos planteados en los proyectos.

\section{CONCLUSIONES}

La metodología propuesta facilitó en gran manera el despliegue de LSS en la empresa seleccionada, ya que brindó los elementos necesarios para diseñar e implementar cambios en la organización que le permitieran desarrollar de forma efectiva proyectos
LSS; identificar las áreas y procesos en donde se debían enfocar las mejoras; identificar proyectos claves; y tener a la mano un procedimiento claro para ejecutar y evaluar los proyectos LSS.

Si bien la implementación generó un impacto positivo en lo financiero y operacional, la evaluación de los resultados del portafolio de proyectos muestra que aún se deben generar cambios fundamentales para lograr mejores resultados, estos cambios son: generar mayor compromiso de la dirección y el personal con la implementación de las acciones propuestas; implementar procesos de capacitación y entrenamiento de los operarios del área de producción; crear políticas y sistemas de incentivos; implementar sistemas de medición de variables claves, lo que elimine la subjetividad en la inspección de los productos; y profundizar sobre el cambio cultural orientado hacia la mejora continua y la excelencia operacional. Como se puede observar en los cambios propuestos, el factor humano juega un papel clave en la implementación de LSS, y más aún en este tipo de organizaciones, cuyos procesos de producción son intensivos en mano de obra.

En general se puede decir que la metodología brinda una base sólida para identificar, definir, priorizar y ejecutar proyectos LSS alineados con la estrategia de la organización, pero a futuro se deben trabajar en algunos aspectos que se deben resolver al momento de definir, implementar y mantener acciones de mejora, entre las cuales se tiene: definir modelos financieros robustos para evaluar y priorizar los proyectos LSS; diseñar estrategias o modelos de cambio organizacional, enfocados en la cultura de mejora Kaizen, ello facilita el compromiso del factor humano y su participación en procesos de mejora por medio de proyectos LSS; diseñar modelos de costos para definir con mayor precisión los ahorros generados por los proyectos LSS; y diseñar modelos de incentivos derivados de los ahorros financieros generados por los proyectos, con el fin de motivar a los empleados a participar en los procesos de mejora $\mathrm{y}$ hacerlos beneficiarios de los resultados obtenidos.

\section{REFERENCIAS}

[1] R.D. Snee. "Lean Six Sigma - getting better all the time". International Journal of Lean Six Sigma. Vol. 1, Issue 1, pp. 9-29. 
March, 2010. ISSN: 2040-4166. DOI: 10.1108/20401461011033130.

[2] J. Antony, A. Kumar and R. Bañuelas "World Class Applications of Six Sigma". Butterworth-Heinemann. 2006.

[3] J.A. Maneesh Kumar. "Does size matter for Six Sigma implementation?: Findings from the survey in UK SMEs". The TQM Journal. Vol. 21, Issue 6, pp. 623-635. February, 2009. ISSN: 1754-2731. DOI: 10.1108/17542730910995882.

[4] A.C. Sunil and V. Deshmukh. "Six Sigma and SMEs: a critical review of literature". International Journal of Lean Six Sigma. Vol. 3, Issue 2, pp. 157-167. January, 2012. ISSN: 2040-4166. DOI: 10.1108/20401461211243720.

[5] J. Antony, M. Kumar and C.N. Madu. "Six sigma in small- and medium-sized UK manufacturing enterprises: Some empirical observations". International Journal of Quality \& Reliability Management. Vol. 22, Issue 8, pp. 860-874. January, 2005. ISSN: 0265671X. DOI: 10.1108/02656710510617265.

[6] M. Kumar and J. Antony. "Comparing the quality management practices in UK SMEs". Industrial Management \& Data Systems. Vol. 108, Issue 9, pp. 1153-1166. October, 2008. ISSN: 0263-5577. DOI: $10.1108 / 02635570810914865$.

[7] W. Timans, J. Antony, K. Ahaus and R. van Solingen. "Implementation of Lean Six Sigma in small- and medium-sized manufacturing enterprises in the Netherlands". Journal of the Operational Research Society. Vol. 63, Issue 3, pp. 339-353. May, 2011. ISSN: 0160-5682. DOI: 10.1057/jors.2011.47.

[8] A. Thomas, R. Barton and C. ChukeOkafor. "Applying lean six sigma in a small engineering company - a model for change". Journal of Manufacturing Technology Management. Vol. 20, Issue 1, pp. 113-129. December, 2008. ISSN: 1741-038X. DOI: 10.1108/17410380910925433.

[9] T. Andrew and R. Barton. "Using the Quick Scan Audit Methodology (QSAM) as a precursor towards successful Lean Six Sigma implementation". International Journal of Lean Six Sigma. Vol. 2, Issue 1, pp. 41-54. March, 2011. ISSN: 2040-4166. DOI: $10.1108 / 20401461111119440$.
[10] M. Kumar, J. Antony and M.K. Tiwari. "Six Sigma implementation framework for SMEs - a roadmap to manage and sustain the change". International Journal of Production Research. Vol. 49, Issue 18, pp. 5449-5467, September, 2011. ISSN: 0020-7543. DOI: 10.1080/00207543.2011.563836.

[11] S.M. Gnanaraj, S.R. Devadasan, R. Murugesh and C.G. Sreenivasa. "Sensitisation of SMEs towards the implementation of Lean Six Sigma - an initialisation in a cylinder frames manufacturing Indian SME”. Production Planning \& Control. Vol. 23, Issue 8, pp. 599608. August, 2012. ISSN: 0953-7287. DOI: 10.1080/09537287.2011.572091.

[12] J. Folaron. "The Evolution of Six Sigma". Six Sigma Forum Magazine. Vol. 2, Issue 4. August, 2003.

[13] J. Maleyeff, E. Arnheiter and V. Venkateswaran. "The continuing evolution of lean six sigma". The TQM Journal. Vol. 24, Issue 6, pp. 542555. September, 2012. ISSN: 1754-2731. DOI: $10.1108 / 17542731211270106$.

[14] M.P.J. Pepper and T.A. Spedding. "The evolution of lean Six Sigma". International Journal of Quality \& Reliability Management. Vol. 27, Issue 2, pp. 138155. January, 2010. ISSN: 0265-671X. DOI: 10.1108/02656711011014276.

[15] T.N. Goh. "A strategic assessment of six sigma". Quality and Reliability Engineering International. Vol. 18, Issue 5, pp. 403-410. August, 2002. ISSN: 1099-1638. DOI: 10.1002/qre.491.

[16] D.C. Montgomery and W.H. Woodall. "An Overview of Six Sigma". International Statistical Review. Vol. 76, Issue 3, pp. 329346. December, 2008. ISSN: 03067734. DOI: 10.1111/j.1751-5823.2008.00061.x.

[17] R.G. Schroeder, K. Linderman, C. Liedtke and A.S. Choo. "Six Sigma: Definition and underlying theory". Journal of Operations Management. Vol. 26, Issue 4, pp. 536-554. July, 2008. ISSN: 02726963. DOI: 10.1016/j. jom.2007.06.007.

[18] D.T. Jones and J.P. Womack. "Lean Thinking: Cómo utilizar el pensamiento Lean para eliminar los despilfarros y crear valor en la empresa". Grupo Planeta. 2012.

[19] T. Pyzdek and P.A. Keller. "The Six Sigma Handbook: A Complete Guide for Green 
Belts, Black Belts, and Managers at All Levels". McGraw-Hill Professional. 2009.

[20] R.B. Pojasek. "Lean, six sigma, and the systems approach: Management initiatives for process improvement". Environmental Quality Management. Vol. 13, Issue 2, pp. 85-92. December, 2003. ISSN: 15206483. DOI: 10.1002/tqem.10113.

[21] E.D. Arnheiter and J. Maleyeff. "The integration of lean management and Six Sigma". The TQM Magazine. Vol. 17, Issue 1, pp. 5-18. February, 2005. ISSN: 0954-478X. DOI: $10.1108 / 09544780510573020$.

[22] J. Antony. "Six Sigma vs Lean: Some perspectives from leading academics and practitioners". International Journal of Productivity and Performance Management. Vol. 60, Issue 2, pp. 185190. January, 2011. ISSN: 1741-0401. DOI: 10.1108/17410401111101494.

[23] R. Andersson, H. Eriksson and H. Torstensson. "Similarities and differences between TQM, six sigma and lean". The TQM Magazine. Vol. 18, Issue 3, pp. 282-296. January, 2006. ISSN: 0954-478X. DOI: 10.1108/09544780610660004.

[24] R.D. Snee and R.W. Hoerl. "Integrating Lean and Six Sigma - a Holistic Approach". Six Sigma Forum Magazine. Vol. 6, Issue 3, pp. 15-21. May, 2007.

[25] M. Assarlind, I. Gremyr and K. Bäckman. "Multi-faceted views on a Lean Six Sigma application". International Journal of Quality \& Reliability Management. Vol. 29, Issue 1, pp. 21-30. June, 2012. ISSN: 0265-671X. DOI: 10.1108/02656711211190855.

[26] R. Shah, A. Chandrasekaran and K. Linderman. "In pursuit of implementation patterns: the context of Lean and Six Sigma". International Journal of Production Research. Vol. 46, Issue 23, pp. 6679-6699. December, 2008. ISSN: 0020-7543. DOI: 10.1080/00207540802230504.

[27] A.J. Thomas and D. Webb. "Quality systems implementation in Welsh small- to mediumsized enterprises: A global comparison and a model for change". Proceedings of the Institution of Mechanical Engineers, Part B: Journal of Engineering Manufacture. Vol. 217, Issue 4, pp. 573-579. April, 2003. ISSN: 09544054. DOI: 10.1243/095440503321628251.
[28] A. Thomas, R. Barton and C. ChukeOkafor. "Applying lean six sigma in a small engineering company - a model for change". Journal of Manufacturing Technology Management. Vol. 20, Issue 1, pp. 113-129. December, 2008. ISSN: 1741-038X. DOI: 10.1108/17410380910925433.

[29] S.S. Chakravorty. "Six Sigma programs: An implementation model". International Journal of Production Economics. Vol. 119, Issue 1, pp. 1-16. May, 2009. ISSN: 09255273. DOI: 10.1016/j.ijpe.2009.01.003.

[30] G. Knowles, L. Whicker, J.H. Femat and F.D.C. Canales. "A conceptual model for the application of Six Sigma methodologies to supply chain improvement". International Journal of Logistics Research and Applications. Vol. 8, Issue 1, pp. 51-65. 2005. ISSN: 13675567. DOI: $10.1080 / 13675560500067459$.

[31] G. Wessel and P. Burcher. "Six sigma for small and medium-sized enterprises". The TQM Magazine. Vol. 16, Issue 4, pp. 264272. January, 2004. ISSN: 0954-478X. DOI: 10.1108/09544780410541918.

[32] P.S. Pande, R.P. Neuman and R.R. Cavanagh "The Six Sigma Way: How GE, Motorola, and Other Top Companies are Honing Their Performance". $1^{a}$ edición. McGraw-Hill. 2000.

[33] R.B. Coronado and J. Antony. "Critical success factors for the successful implementation of six sigma projects in organisations". The TQM Magazine. Vol. 14, Issue 2, pp. 92-99. April, 2002. ISSN: 0954-478X. DOI: $10.1108 / 09544780210416702$.

[34] A. Chakraborty and K. Chuan. "Qualitative and Quantitative Analysis of Six Sigma in Service Organizations". In Total Quality Management and Six Sigma, T. Aized. Ed. InTech. 2012.

[35] A.C. Laraia, P.E. Moody and R.W. Hall. "The Kaizen Blitz: Accelerating Breakthroughs in Productivity and Performance". Wiley. 1999.

[36] K. Linderman, R.G. Schroeder, S. Zaheer and A.S. Choo. "Six Sigma: a goal-theoretic perspective". Vol. 21, Issue 2, pp. 193-203. March, 2003. ISSN: 0272-6963. DOI: 10.1016/S0272-6963(02)00087-6.

[37] K.M. Henderson and J.R. Evans. "Successful implementation of Six Sigma: benchmarking General Electric Company”. Benchmarking: 
An International Journal. Vol. 7, Issue 4, pp. 260-282. October, 2000. ISSN: 14635771. DOI: $10.1108 / 14635770010378909$.

[38] J. Antony and R. Banuelas. "Key ingredients for the effective implementation of Six Sigma program". Measuring Business Excellence. Vol. 6, Issue 4, pp. 20-27. December, 2002. ISSN: 1368-3047. DOI: 10.1108/13683040210451679.

[39] K.Yang. "Voice of the Customer: Capture and Analysis". McGraw-Hill Professional. 2007.

[40] S. Karthi, S.R. Devadasan and R. Murugesh. "Integration of Lean Six-Sigma with ISO 9001:2008 standard". International Journal of Lean Six Sigma. Vol. 2, Issue 4, pp. 309-
331. November, 2011. ISSN: 2040-4166. DOI: $10.1108 / 20401461111189416$.

[41] A. Larson. "Demystifying Six Sigma: A Company-wide Approach to Continuous Improvement". AMACOM. 2003.

[42] G. Brue. "Six Sigma for Small Business". Entrepreneur Press. 2006.

[43] M.M. Fernandes, M.B. Da Silva and J.B. Turrioni. "Modelo teórico-conceitual para gestão de portfólio de projetos Seis Sigma". Revista de Gestão e Projetos. Vol. 1 N $^{\circ} 2$, pp. 3-25. Mayo 2011. ISSN: 2236-0972. DOI: $10.5585 /$ gep.v1i2.13.

[44] D.C. Montgomery "Introduction to statistical quality control”. Wiley. Hoboken, NJ, USA. 2009. 